\title{
Correlation of Thermo-Elastic Material and Corrosion Behavior of Refractory Castables by In-Situ Measurements
}

\author{
Wanja Reichert ${ }^{1, * \mathbb{D}}$, Jonas Nießen ${ }^{1, *}$, Peter Leto ${ }^{2}$, Simon Etzold ${ }^{1}$, Eva Kröll ${ }^{1}$, \\ Thorsten Tonnesen ${ }^{1}$ and Rainer Telle ${ }^{1}$ \\ 1 Institute of Mineral Engineering, RWTH, 52066 Aachen, Germany; etzold@ghi.rwth-aachen.de (S.E.); \\ eva.kroell@live.de (E.K.); tonnesen@ghi.rwth-aachen.de (T.T.); telle@ghi.rwth-aachen.de (R.T.) \\ 2 Institute of Chemical Technologies and Analytics, 1060 Vienna, Austria; ptrleto@gmail.com \\ * Correspondence: reichert@ghi.rwth-aachen.de (W.R.); j.niessen@ghi.rwth-aachen.de (J.N.); \\ Tel.: +49-241-80-98324 (W.R.); +49-241-80-97705 (J.N.)
}

Received: 15 January 2020; Accepted: 4 March 2020; Published: 9 March 2020

\begin{abstract}
Corrosion is one of the most common wear mechanisms of refractories. Corrosive attacks lead to chemical and microstructural changes. Hot corrosion compromises chemical and/or physical interactions. Thus, the process is complex and not yet fully understood. Currently, corrosion is investigated post mortem by means of X-ray diffraction or scanning electron microscopy. These methods have the drawback that some information is lost on cooling. In-situ measurements, however, take measurements within the process. In resonant frequency and damping analysis (RFDA), a sample is excited to vibrate by a mechanical impulse. The vibrating sample emits an acoustic signal. This is recorded with a microphone and evaluated by means of Fast Fourier Transformation (FFT). We measured the change of the frequency of a low cement castable during the corrosion process. Further simplified experiments with less complex materials were done to confirm the results. Distinctive points of the curves could be correlated to specific corrosion phenomena, like melting or infiltration. The applied methods include a first characterization of the material with open porosity, density and in-situ high-temperature (HT)-RFDA measurements as well as a study of the slag behavior.
\end{abstract}

Keywords: impulse excitation technique (IET); corrosion; elastic properties; in-situ corrosion measurement; blast furnace slag

\section{Introduction}

Refractory materials are most suitable and developed for extreme operating conditions. In addition to high temperatures, the refractory linings are also often exposed to highly corrosive media. The different chemical compositions of material and corrosion medium leads to a gradient of the chemical potential. This makes interactions between these two partners unavoidable. The kinetics of the reactions is favored at high temperatures. In addition to the chemical attack, the material can also be subjected to mechanical stress. Stress can for example occur due to reaction based phase transformation, that may result in a mismatching the coefficient of thermal expansion [1]. The progress of the corrosion process can lead to phase and microstructural changes in the material. If the refractory materials are not precisely tailored to the given process parameters, above-average wear may occur. This results in immense economic damage. Due to the chemical and physical interactions, combined with the heterogeneous material behavior, the prediction of the corrosion process, especially with industrial melts and slags, is extremely complex. Predicting the behavior of refractories under corrosive attack is 
not a simple task since refractories are heterogeneous multicomponent solids varying in crystallinity, porosity, purity, grain size distribution and morphology.

There are several ways to describe refractory corrosion-utilizing acid-base theory, thermodynamics and kinetics. In conclusion, corrosion of refractories is more of a chemical nature, with higher rates at elevated temperatures as the reactivity of solids increase. Whether it is a solid versus solid reaction, where a liquid is formed at high temperatures, or a solid-fluid interaction, fluid mass transportability by convection or diffusion can substantially enhance the corrosion rate. Thus, kinetic phenomena accompanying these reactions are worth considering as well. Most important kinetic factors influencing corrosion include penetration, dissolution and spalling [2].

Currently, corrosion phenomena are mainly investigated by post mortem methods [3]. The samples are subjected to static or dynamic tests under simulated test conditions. Subsequently, the mineralogical composition and microstructural changes of the specimens are examined by X-ray diffraction (XRD) and scanning electron microscopy (SEM). However, post mortem examination has some disadvantages. Phase transitions may occur during cooling. Therefore, the phase composition at room temperature can deviate significantly from the composition under operating conditions. This makes precise analysis more difficult. Furthermore, the kinetics cannot be determined exactly. The speed of the entire reaction can only be estimated using a complex series of tests. Statements about individual partial reactions are only possible in individual cases. The possibility to track the corrosion process in-situ offers great possibilities to better understand the complicated corrosion processes. Moreover, important information on kinetics can also be obtained. Based on new findings, the material design can be adapted and optimized.

Mechanical properties evaluation of refractory materials is of high interest, since not only mechanical forces, but also corrosion can induce irreversible damage to the material, which ultimately leads to mechanical failure, such as fracture. Routinely conducted bend or flexural strength tests (three-point and four-point bending flexural tests) revealing Hooke's law proportionality, from which one can also draw conclusions on the fracture toughness of the material, are also applicable to high-temperatures. However, they do not allow an in-situ evaluation of elastic properties during heating or cooling, respectively [4].

By means of resonant frequency and damping analysis (RFDA), the change of the elastic properties during the corrosion process shall be recorded in-situ. According to [5], the frequency $f$ is related to the Young's modulus $E$ by the equation,

$$
E=C_{1} m f^{2}
$$

whereas $C_{1}$ is determined by geometrical factors and $\mathrm{m}$ represents the mass of the body. High-temperature resonant frequency and damping analysis (HT-RFDA) test facilities are able to fulfill this task and conduct an in-situ measurement of material elastic properties at high temperatures. Moreover, in contrast to three-point bending tests, they are of a non-destructive nature. This should provide additional information to better understand and predict the process in general. During the RFDA test, a sample is excited to vibrate by a slight mechanical impulse. The sample vibrates and emits an acoustic signal. This is recorded with a microphone and evaluated by means of Fast Fourier Transformation (FFT). The FFT transforms the time pendent acoustic signal into its constituent frequencies. In the case of pure elastic behavior and a rectangular rod, the recorded resonant frequency can be converted into the elastic parameters according to ASTM C1548-02 [5]. As already mentioned, corrosion leads to a chemical and microstructural change in the material. The elastic properties correlate directly with the phase composition and the microstructure [1]. Thus, the corrosion progress must be detectable with the RFDA. There are several previous attempts to examine whether this monitoring of processes such as sintering [6], phase transitions [7,8], thermoshock and fatigue [9,10], effects of additives [11,12] or corrosion [13-15] with aid of the RFDA is possible. Previous studies could not explain the measured frequency change with known corrosion phenomena. The aim of this work is to find a direct correlation such as the melting of the slag and the influence on the frequency. For this 
purpose, we investigated different materials and filler combinations in order to examine individual aspects isolated.

\section{Materials and Methods}

\subsection{Materials}

Materials chosen for the investigation are a dense aluminum oxide ceramic known as alumina, a tabular alumina-based low cement castable (LCC) and aluminum. The alumina bars were isostatically pressed from $99.98 \%$ alumina powder. The LCC were produced in the laboratory from tabular alumina in different grain sizes together with a deflocculent, reactive alumina and cement. Formulation and further details can be found in [13]. All samples were sintered prior to the corrosion tests. For the frequency measurements, bars of $25 \times 25 \times 150 \mathrm{~mm}^{3}$ were produced. Furthermore, in some of the samples, cavities of $\left(7 \times 12 \times 40 \mathrm{~mm}^{3}\right)$ are introduced into the green bodies by a milling machine. Sintering Temperatures are $1500{ }^{\circ} \mathrm{C}$ for the $\mathrm{LCC}$ and $1600{ }^{\circ} \mathrm{C}$ for the $\mathrm{Al}_{2} \mathrm{O}_{3}$-ceramic, respectively.

Materials added to the cavity are water (incl. ice) and oil as liquids, gallium and field's metal (eutectic alloy of In, Bi and Sn) as metals and a blast furnace slag as a corrosive medium. Table 1 shows the different sample and filler combinations investigated in this study. The interaction of LCC and slag are the main subject of this study. Due to the high complexity based on heterogeneous material behavior and a multi-component slag, simpler material combinations were tested. Aluminum and alumina were chosen to yield homogeneous material behavior and different elastic properties. Water and rap oil were used as fillers to study the general influence of different viscous fluids on the frequency at room temperature. Ice, Gallium and field's metal were used to investigate the frequency change during a phase transition. Different combinations of sample materials and fillers were tested to investigate possible interactions of their characteristics.

Table 1. Experimental matrix: combinations of material with fillers.

\begin{tabular}{ccccccc}
\hline Sample & \multicolumn{7}{c}{ Filler } \\
\cline { 2 - 7 } Material & Water & Rap Oil & Ice & Gallium & Field's Metal & Slag \\
\hline Aluminum & $\mathrm{X}$ & $\mathrm{X}$ & $\mathrm{X}$ & & & \\
Alumina & $\mathrm{X}$ & $\mathrm{X}$ & $\mathrm{X}$ & $\mathrm{X}$ & $\mathrm{X}$ & $\mathrm{X}$ \\
LCC & $\mathrm{X}$ & $\mathrm{X}$ & & $\mathrm{X}$ & & $\mathrm{X}$ \\
\hline
\end{tabular}

\subsection{Measurements}

The following software parameters are given in order to reproduce the data obtained in this study. The elastic properties were analyzed by two different systems from IMCE (Genk, Belgium), the RFDA System 23 and RFDA-HT1750. The data acquisition time after each excitation was set to $0.350 \mathrm{~s}$. To sort out undesired frequencies, a high pass filter was activated with a low cut-off frequency of $1000 \mathrm{~Hz}$. The power of the excitation magnet was adjusted to $30 \%$ of the maximum impulse strength. The number of analyzed frequencies for every measurement was set to eight. The Fourier spectrum was regulated by adjusting the signal noise percentage of the maximum amplitude (15\%) and by setting its resolution, which was achieved by adjustment of the maximum number of points (frequency steps) from the signal for Fast Fourier Transformation (FFT) calculation (65,536 points). In compliance with ASTM E 1876, consequences of high-temperature exposure combined with a corrosive attack were detected by means of the HT-RFDA measuring device. In this device, a furnace can be placed above two sample holders, each equipped with a mechanical actuator. The signal is transferred to the microphone via a waveguide tube above the sample as displayed in Figure 1. Next to the tube, a thermocouple is placed 4-6 mm above the sample for temperature measurements. 


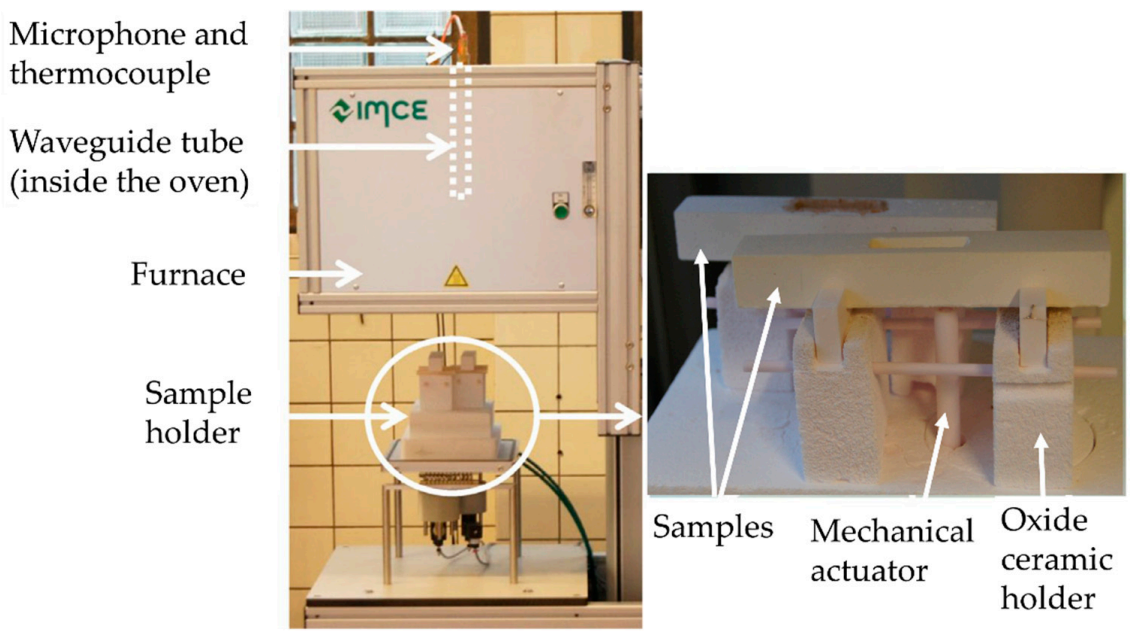

Figure 1. RFDA facility for high temperature investigation. RFDA, Resonant Frequency Damping Analysis [16].

Long-term high-temperature exposure of $12 \mathrm{~h}$ at $1400{ }^{\circ} \mathrm{C}$ was chosen to simulate the operating conditions. Each sample was excited every $30 \mathrm{~s}$ during heating with a rate of $2{ }^{\circ} \mathrm{C} / \mathrm{min}$, to provide a sufficient amount of available data even during occurring material changes.

As the HT-RFDA device is most suitable for the examination of isotropic, homogeneous materials with simple geometry, there are some obstacles when it comes to complex dimensions and anisotropic materials with multiphase composition. The above-mentioned elastic constants react to changes of microstructure (phase formation, crack initiation and propagation, thermal stresses due to thermal expansion, melting, recrystallization, etc.) and further rely on other parameters such as temperature or atmospheric pressure. Thus, the evaluation of material specific resonant frequencies constitutes a powerful tool for material characterization [17].

The sample can be positioned on different types of supports, which consequently become nodes of well-defined vibration modes [7], while the impact spot should be near an antinode of the induced vibration [17]. The position of each of the depicted nodes results from an effort to minimize energy losses caused by friction [18]. To enable free vibration of the sample in the fundamental transverse mode, the positions exhibiting no displacement (nodal points) are located at $0.224 \times \mathrm{L}$ from each end, where L represents the sample length [5]. In this study, the measurements are conducted in flexural mode. The recorded time-dependent signal is then transformed into a frequency dependent $(\mathrm{fr})$ data by Fast Fourier Transformation (FFT).

It is important to point out that the HT-RFDA software cannot calculate elastic properties from frequency data as the geometry deviates from a rectangular shape due to the intrusion of a cavity. These cavities enabled the insertion of the corrosive medium. Moreover, the software does not consider the deviation from pure elastic behavior because of the melted slag at elevated temperatures. Additionally, testings of these samples should show the influence of a cavity on the detected frequency due to the overall change of the sample geometry.

After corrosion, post mortem analysis was performed to correlate the method with standard tests. X-ray diffraction (XRD) method was applied using a Bruker D8 Advance with LynxEye detector (Bruker AXS, Karlsruhe, Germany), CuK $\alpha$ tube and nickel filter, in order to investigate the mineralogical composition. The chosen parameters include a measurement range from $5-90^{\circ} 2 \theta$ in 0.01 steps at $0.5 \mathrm{~s}$ per step. Microstructural changes were investigated via scanning electron microscopy (SEM) with LEO Type 440i (Leo Electron Microscopy, Cambridge, UK).

\subsection{Corrosive Medium}

To estimate the material behavior in operating condition, a blast furnace slag was inserted into the cavity of the samples. Before the X-ray fluorescence (XRF) measurement, using a PW2404 device 
(Malvern PANalytical B.V., Eindhoven, The Netherlands), the given mineral samples were fused to glass disks by a Claisse LeNeo instrument (Malvern PANalytical B.V., Eindhoven, The Netherlands). Analysis revealed the slag composition, which mostly consists of $\mathrm{CaO}, \mathrm{SiO}_{2}$ and $\mathrm{Al}_{2} \mathrm{O}_{3}$ (in descending order of weight percent) and some minor portions of other oxides regarded as slag impurities. The exact composition is shown in Table 2. The blast furnace slag is of a basic nature exhibiting a C:S ratio of approximately 5:3.

Table 2. Composition of the blast furnace slag used in the corrosion tests ${ }^{1}$.

\begin{tabular}{cc}
\hline Component & Portion [wt.-\%] \\
\hline $\mathrm{CaO}$ & 47.0 \\
$\mathrm{SiO}_{2}$ & 32.4 \\
$\mathrm{Al}_{2} \mathrm{O}_{3}$ & 10.1 \\
$\mathrm{MgO}$ & 5.5 \\
$\mathrm{SO}_{3}$ & 2.5 \\
$\mathrm{TiO}_{2}$ & 1.1 \\
$\mathrm{~K}_{2} \mathrm{O}$ & 0.5 \\
$\mathrm{MnO}$ & 0.4 \\
$\mathrm{Fe}_{2} \mathrm{O}_{3}$ & 0.3 \\
$\mathrm{Na}_{2} \mathrm{O}$ & 0.2 \\
\hline $\mathrm{LoI}\left(1050{ }^{\circ} \mathrm{C}\right)$ & -1.89 \\
\hline
\end{tabular}

\section{Results}

\subsection{Procedure}

As already stated, previous works studied the in-situ monitoring of the corrosion process with the help of the RFDA technique [13-15]. However, the development of the recorded frequency signals could not be clearly assigned to the phenomena occurring during corrosion. We therefore decided to examine fundamental aspects such as melting on simplified systems. Hence, we performed several basic experiments prior to the corrosion test of the LCC to explain certain phenomena that occur within the process. To reduce the amount of uncertainties arising from heterogeneous material behavior, we chose to perform the preliminary results with alumina and aluminum samples. Moreover, we decided to perform the tests near room temperature, as the signal strength received via RFDA decreases with increasing temperature. We also chose clearly defined materials, such as eutectic alloys, to fill the cavity and examine the interaction between sample and added medium.

The previous work of Sibil et al. [7] has shown that the signal quality in the high temperature range is strongly impaired. Therefore, we have decided to use fillers that already melt below $100^{\circ} \mathrm{C}$. In the past, the poor signal quality at rising temperatures leads to gaps and unexplainable jumps when plotting frequency versus temperature. Due to the relatively poor signal quality, the most prominent frequency in the acoustic signal fluctuates with every single measurement, which makes the automatic identification of the correct frequency by the software questionable. Consequently, we have decided to record up to eight frequencies per measurement and evaluate all recorded data. This not only allows the tracking of the main frequency, but also additional frequencies, which might also include information supporting the identification of discontinuities, related to the material behavior. Larger deviations can usually be identified as inaccurate measurements.

\subsection{Fundamental, Simplified Investigations}

\subsubsection{Influence of the Cavity}

In the case of a rectangular rod, the recorded resonant frequency can be converted into the elastic parameters according to ASTM C1548-02 [5]. A notch, submitted into the sample allowed the addition 
of a corrosive agent. Due to the change in geometry, the calculation of the elastic properties according to the standard is not valid anymore.

For a better understanding of the influence of the cavity, we performed measurements of several different samples, both with and without inserted corrosive medium as well as with different material combinations. The comparison of the two different geometries shows that introducing the cavity reduces the main frequency independent of the measured material. The relative change of frequency against temperature however, is comparable. In previous investigations [13] you can get more detailed information on these issues. Therefore, any distinctive curve shapes or curve fluctuations of samples with a milled cavity plotted in the following high-temperature tests will not be regarded as a result of changed geometry or stresses induced during milling of cavities.

\subsubsection{Influence of Liquids and Solids in the Cavity}

We measured the frequency of all three mentioned materials: The LCC, alumina and aluminum. For all samples with milled cavity, we performed measurements before and after filling the cavity stepwise with either water or rap oil up to a maximum of $3 \mathrm{~mL}$. Increasing the amount of liquids in the mold result in a decrease in the frequency. However, the effect was rather small with a relative change of less than $1 \%$ in frequency. Additionally, there was no major difference between the addition of oil or water.

In further tests, we examined the frequency change during a phase transformation from solid to liquid. Therefore, water was casted into the cavity and kept at $-20{ }^{\circ} \mathrm{C}$ until it was completely frozen. During measurements at ambient temperature, the temperature of the sample continually increased. The frequency was recorded every $10 \mathrm{~s}$. In parallel, the values for a sample without notch are determined at the same temperature. As the furnace had to be shut for the measurement, both tests were stopped, when the frequency did not change anymore. Figure 2 reveals that there is a sudden drop in the frequency of the samples filled with ice around $4.5 \mathrm{~min}$. Surprisingly, there was still ice remaining in the cavity after $7.5 \mathrm{~min}$, even though the frequency was constant. This and the abrupt change of frequency indicates that the first occurrence of a liquid phase has a strong impact on the elastic response. Further melting has just a minor or no impact on the detected frequency. This behavior might be related to the wetting of the interface between vessel and ice.

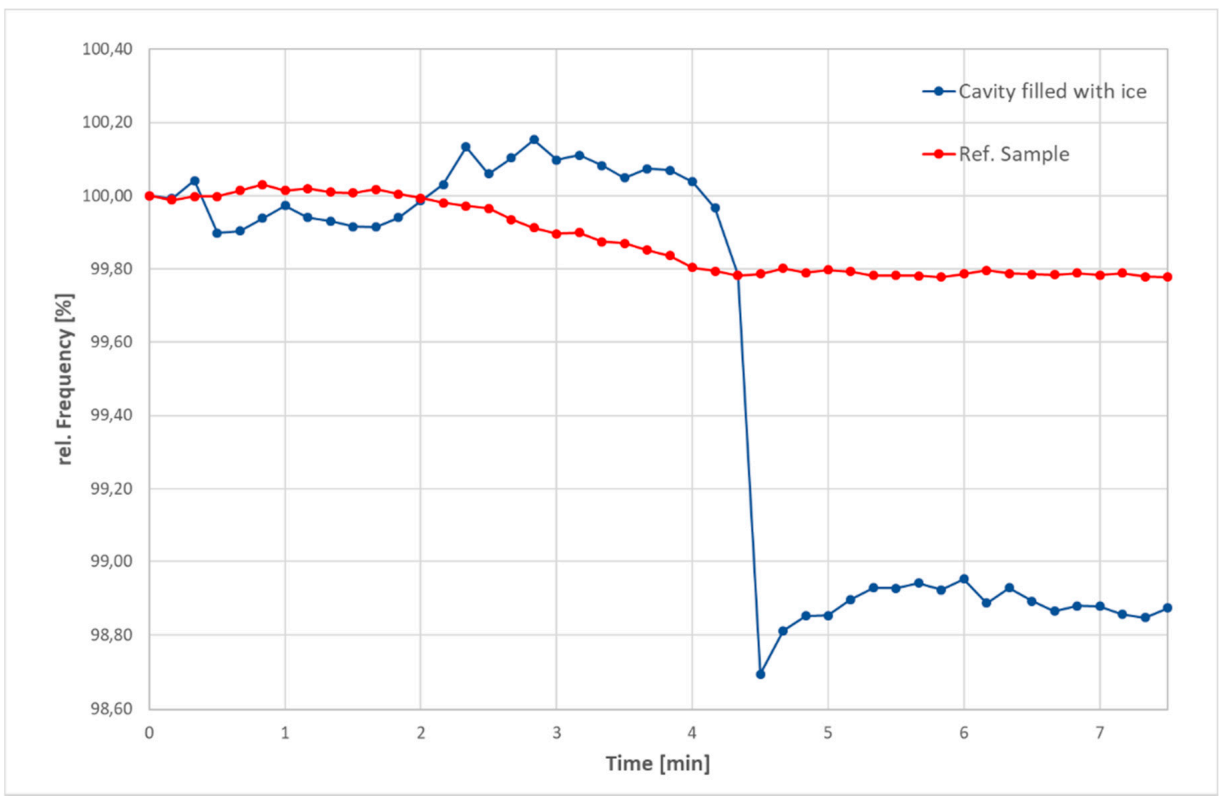

Figure 2. Relative change of frequency during melting of ice in the cavity (blue) in comparison with sample without ice (red). 
In order to verify the previous results, we performed additional tests using a eutectic alloy to fill the mold (Figure 3), known as field's metal (with a melting point around $62^{\circ} \mathrm{C}$ ) [19]. The sample was repeatedly cycled between room temperature and $100{ }^{\circ} \mathrm{C}$ with a heating rate of $2{ }^{\circ} \mathrm{C} / \mathrm{min}$. Several iterations show two sharp drops in frequency during the heating phase. The first one is located, despite small deviation, at the melting point of the field's metal around $60{ }^{\circ} \mathrm{C}$. Therefore, this change of frequency can be interpreted as a solid-liquid transition of the metal. The second drop occurs around $85^{\circ} \mathrm{C}$.

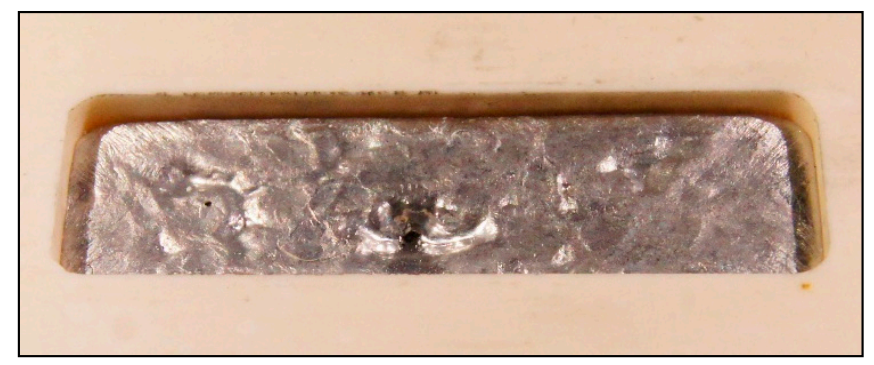

Figure 3. The cavity of the alumina sample filled with field's metal after solidification.

\subsubsection{Difference of Casting and Granular Filling of the Cavity}

The flexural frequency of the sample did not change with inserting slag (loose coarse powder) into the mold. Filling the cavity with small beads of field's metal did not show any major change as well. In contrast to that, the frequency changed notably when liquid metal was casted into the mold and measured after solidification. Addition of field's metal or gallium to alumina resulted in a distinct decrease. We assume that the bond between metal and vessel, displayed in Figure 3, leads to a coupled oscillation behavior. According to Salmang et al. [1], the Young' modulus of a multiphase material lies between the values of the pure components $E_{i}$ and depends on the volume proportions $V_{i}$ and the shape of the components.

\subsection{Corrosion with Blast Furnace Slag}

With the addition of slag (loose coarse powder) into the cavity, the corrosion behavior was investigated. Reichert et al. [13] reported a softening temperature of $1289{ }^{\circ} \mathrm{C}$ and a hemisphere temperature of $1334^{\circ} \mathrm{C}$, determined with hot stage microscopy. A holding temperature at $1400{ }^{\circ} \mathrm{C}$ was chosen. Holding time was set to $12 \mathrm{~h}$ to simulate the operating conditions. In Figure 4 , the LCC and alumina samples are displayed after the high temperature corrosion test. On the right, the cross-section is visible. The grey lines show the original size of the cavity whereas the red lines indicates the infiltration depth. It varies from approx. $4.5 \mathrm{~mm}$ in the LCC to $1.5 \mathrm{~mm}$ in the alumina sample implying $30 \%$ higher infiltration into the LCC material.

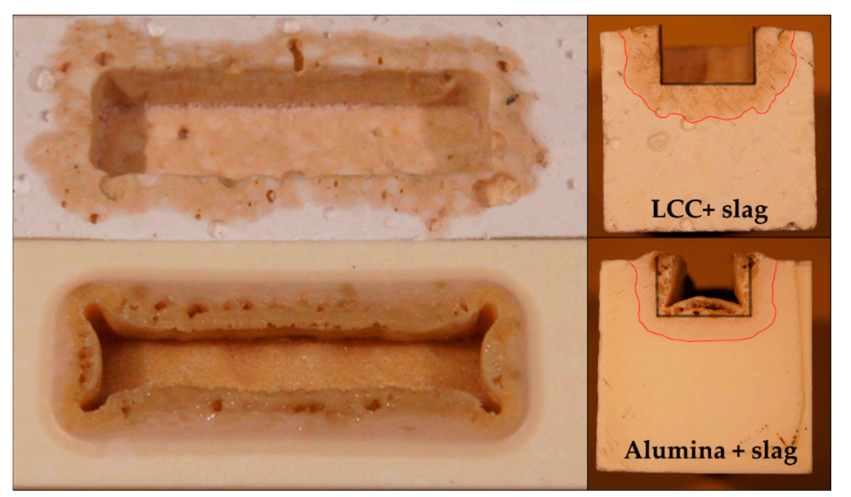

Figure 4. Comparison of the corroded area of the low cement castable (LCC) (top) and alumina (bottom) samples after corrosion test with slag. 
The graphs depicted in Figure 5 show the relative change of frequency with temperature. Increasing the temperature generally leads to a decrease of Young's modulus and therefore to a steady decrease in all graphs [1]. Notable differences from the LCC samples without slag are sudden small drops in frequency evolution at $800{ }^{\circ} \mathrm{C}$. With regard to the results of Section 3.2.3, we did a dilatometry measurement (Linseis L70/2170, Selb, Germany), as forming a bond between slag and sample due to sintering might cause the drop. The dialometry graphs indicate the start of densification around $750{ }^{\circ} \mathrm{C}$. Taking into account that the slag was milled to grain sizes smaller than $63 \mu \mathrm{m}$ prior to the dilatometry the start of densification seems to match the drop in frequency.

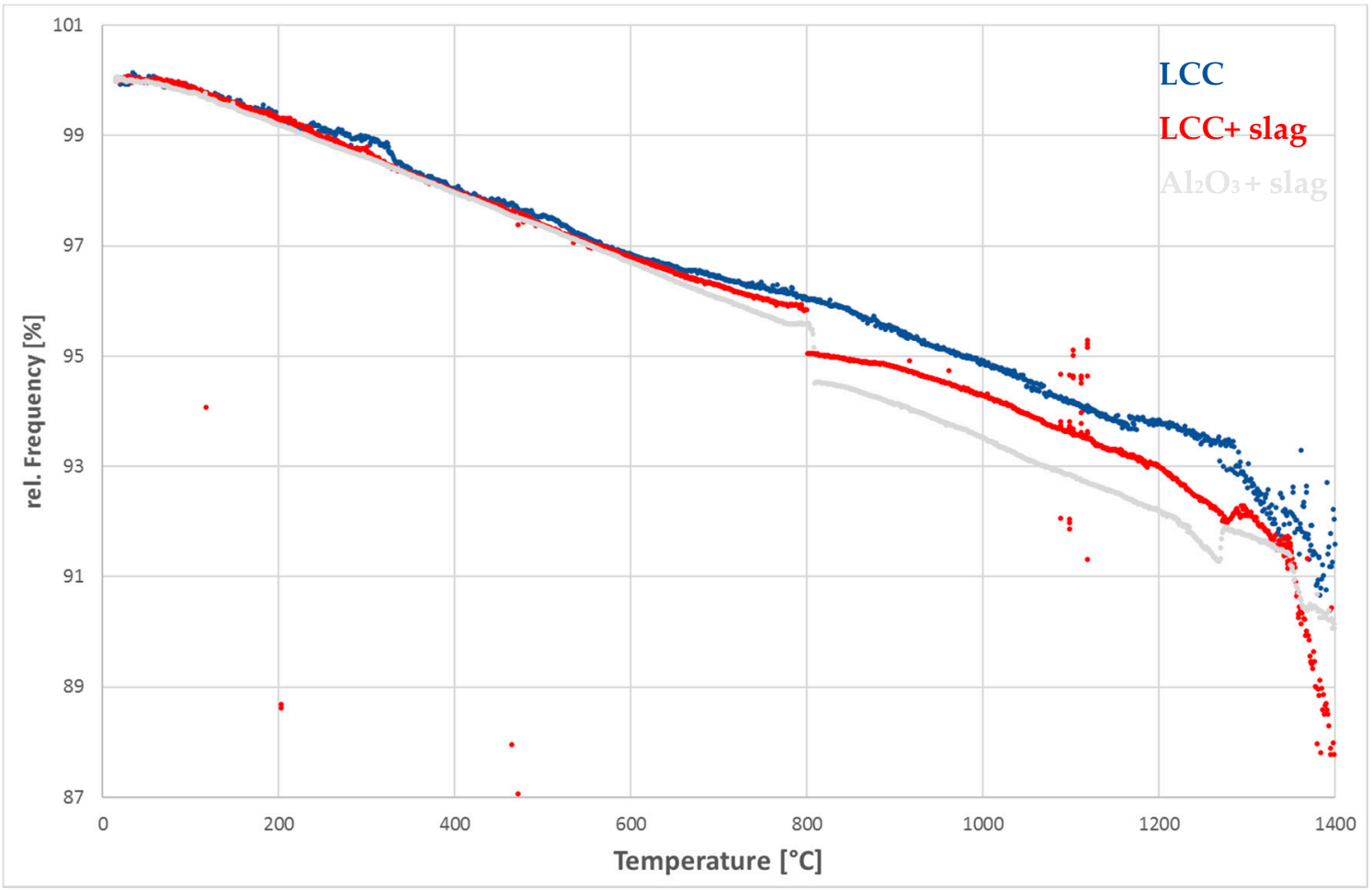

Figure 5. Relative change of frequency against temperature during heating of the reference LCC, the LCC and alumina sample filled with slag.

Around $1250{ }^{\circ} \mathrm{C}$ the samples filled with slag show a small increase of frequency, which is more pronounced in the alumina sample. This particular behavior cannot be explained so far. Around $1350{ }^{\circ} \mathrm{C}$ the curves with slag show a kink and a stronger decrease of frequency than the reference sample. The well-pronounced kink could represent the beginning of the slag's softening or melting. The measured temperature is $35^{\circ} \mathrm{C}$ higher than the one predicated via hot stage microscopy. This difference can be explained by the mismatch in temperature of the sample and the furnace or different particle sizes in the two different experiments. In contrast to the previous results (Section 3.2.2), there is no sudden drop in frequency. The preliminary tests were done with a pure substance and eutectic alloy. These systems have a defined melting point, in contrast to the expectable melting interval of the slag. Another reason for this particular behavior might be the very high viscosity of silicate rich melts near the melting point [20]. In contrast to that, metals and water exhibit a comparably low viscosity. Under the assumption, that a highly viscous fluid shows an oscillation comparable to a solid body, there would be a smaller frequency drop at the melting point.

At about $1370{ }^{\circ} \mathrm{C}$, the frequency evolution of alumina shows another kink. The melting process of the slag is probably finished at that temperature. We assume that after melting, the corrosion of the material begins. The very low corrosion rate of dense alumina is probably also associated with a small change in frequency. In contrast, the slope of the LCC does not change noticeably so the decrease of 
frequency continuous at a high rate. We presume that the reaction rate is higher in the LCC sample because of the much higher surface related to the open porosity.

Curves recorded in the isothermal period (Figure 6, the frequency is plotted over time), exhibit measurement deviations. There is a more or less continuous curve visible for the alumina sample filled with slag. After four hours, the frequency reaches a steady-state and remains constant for the rest of the isothermal time. The steady decrease in all corroded samples indicates that these trends in curve progression are not only a statistical measurement deviation between the curves.

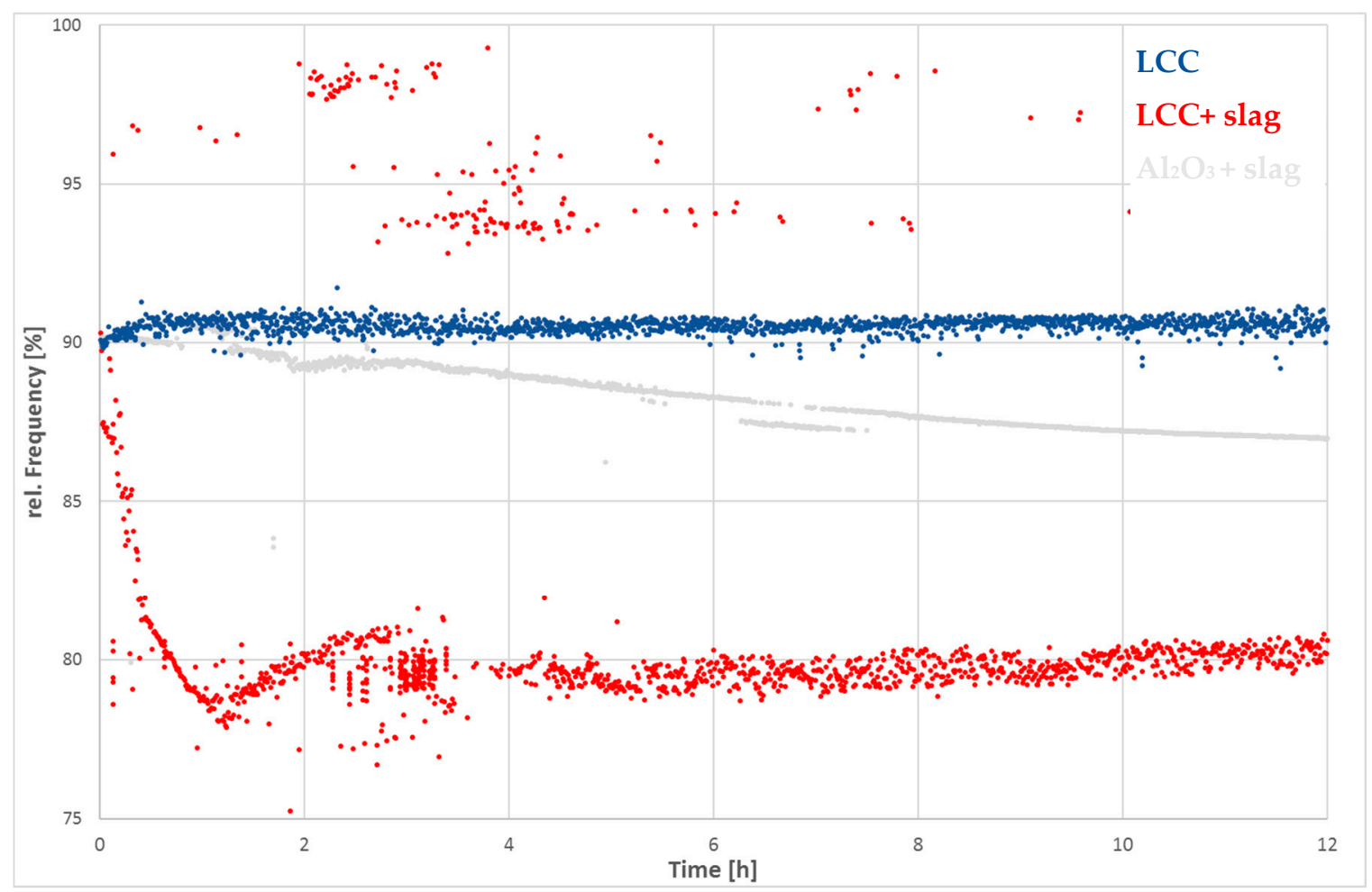

Figure 6. Relative change of frequency against time during the isothermal period of the reference LCC, the LCC and Alumina sample filled with slag, depicting different time-dependent corrosion behavior.

In contrast, the deterioration rate of the alumina curve is smaller. A steady state cannot be unambiguously identified. The slope however, seems to decrease continuously. The steady decrease of the frequency relates to the corrosion reaction of slag and container. The difference of specific surface between the dense alumina sample and LCC seems to be a good explanation for the different time dependent behavior. The slag probably infiltrates the castable and therefore can access a higher reaction surface compared to the alumina bar. On the other hand, the results of Section 3.5 show that the slag preferably reacts with the matrix phase of the $\mathrm{LCC}$, which is composed of calcium-hexa-aluminate $\left(\mathrm{CA}_{6}\right)$. Another difference, compared to the alumina sample, is that the decrease of measured frequencies is distinctively higher due to the faster infiltration and higher corrosion rate.

\subsection{Corrosion Results (XRD)}

The phase compositions of the reference LCC and the corroded sample were evaluated by qualitative powder XRD (Figure 7).

The XRD measurement shows the formation of gehlenite as the main reaction product. Phases known from the uncorroded samples-corundum and $\mathrm{CA}_{6}$ - were detected in relatively high amounts via quantitative $\mathrm{XRD}$ analysis compared to newly generated gehlenite, whose main peak is located at $2 \theta=31.415^{\circ}$. Additionally, $\beta$-alumina was detected in minor quantities as well, coming probably from sodium residues from the Bayer process. The quantitative evaluation showed that the majority 
of the corroded area was indeed composed of the initial phases with corundum and $\mathrm{CA}_{6}$ with small gehlenite content.

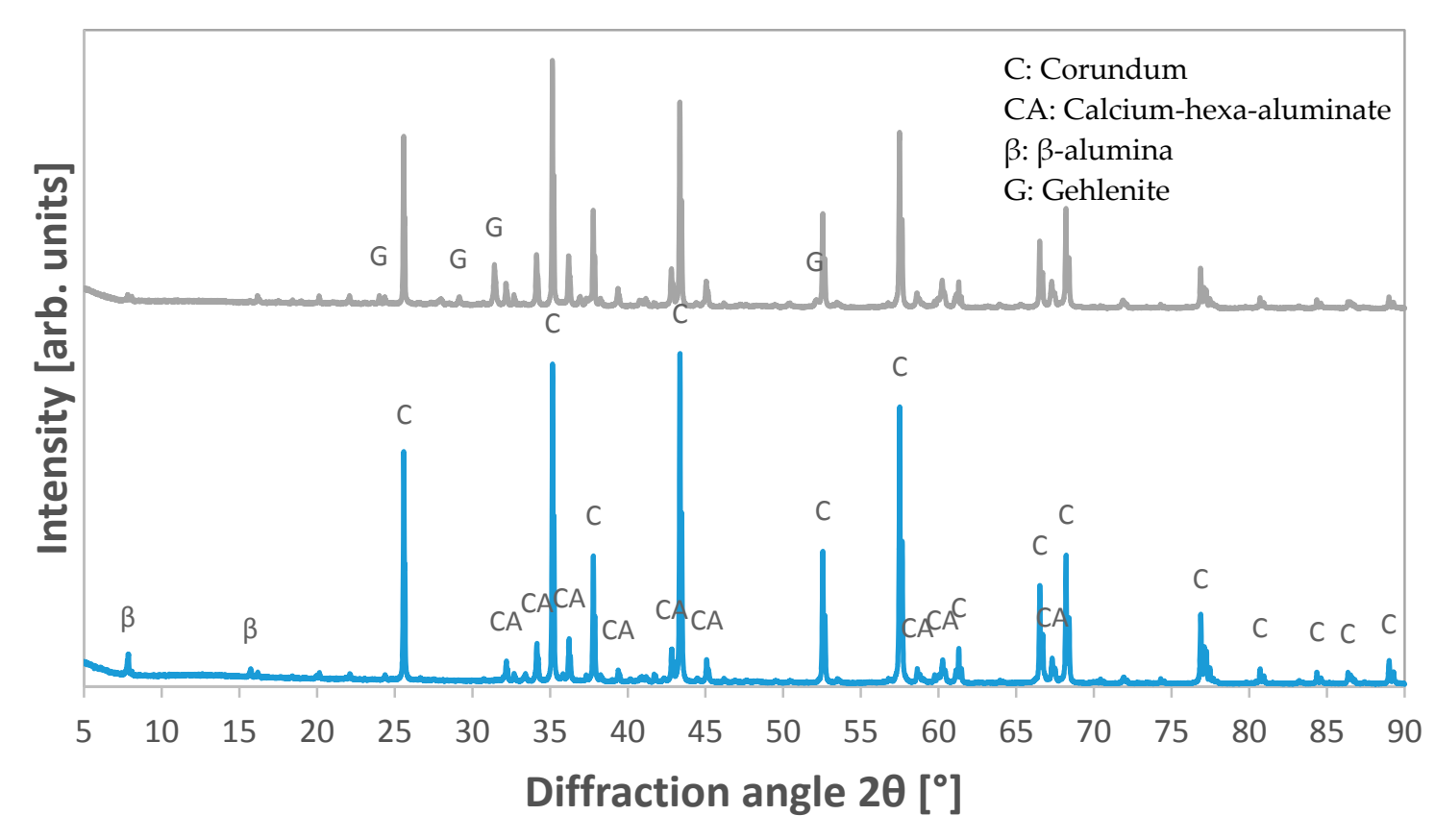

Figure 7. X-ray diffraction (XRD)-plot of the reference LCC (bottom) and the LCC after corrosion with blast furnace slag (top) showing gehlenite as the main reaction product.

\subsection{Corrosion Results (SEM)}

An overview of the two most peculiar zones, the corroded and uncorroded zone in combination with the transient zone of the LCC sample is displayed in Figure 8. In (b) it can be noticed that corrosion leads, compared to the uncorroded zone in (c), to an increased porosity reflected in a distinctively higher number of small pores. The microstructure of the uncorroded zone remained unaltered by the thermal cycle, exhibiting relatively few small-sized production-related pores. The contrast in the backscattered image clearly shows the infiltration of the matrix by the slag. Coarser alumina grains show a thin reaction shell on their surface. However, the impact of the corrosive attack seems to be quite small. Therefore, we conclude that the previously shown formation of gehlenite is a result of a reaction between the slag and the matrix.

As a result, the destructive effect of corrosion caused the formation of a high number of cracks within the bonding phase, which are not present in the uncorroded zone at all. The largest cracks usually proceed closer to the larger alumina grains whereas the smaller cracks progress throughout the matrix. The apparent effect of larger cracks bordering around the big alumina grains indicates interfacial debonding, which denotes detachment of aggregate grains from the matrix. The changeover between these parts in (a) is not abrupt, the size of voids, mostly formed in very close proximity to alumina grains, is continuously decreasing. These microstructural changes are also reflected in the macroscopic appearance (Figure 3). There are bulges, which are related to an increase in volume. Furthermore, macroscopic cracks are visible in the corrosion zone. 

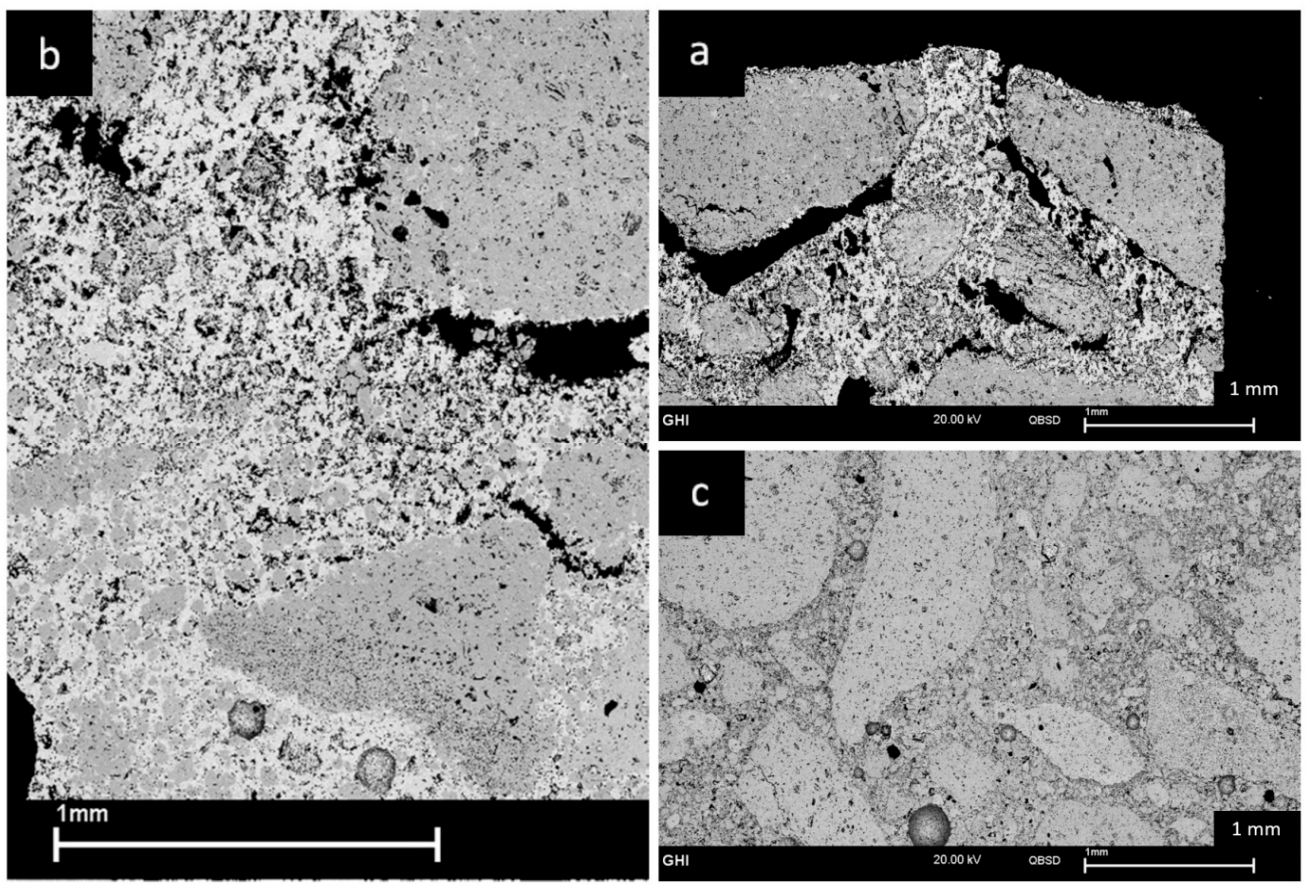

Figure 8. Backscattered SEM image of (a) corroded Zone, (b) infiltrated matrix, (c) uncorroded zone of the LCC sample after corrosion with blast furnace slag, revealing the infiltration, reaction and debonding mechanisms.

\section{Discussion}

The aim of this work was to investigate the corrosion process using RFDA and to correlate the measurement curves with specific corrosion mechanisms. The reliability of the measured values is of particular importance. As can be seen in Figures 5 and 6 , the measured values vary especially in the high temperature range. According to Sibil et al. [7], the attenuation increases with rising temperature due to the softening of glass phases. This affects the signal and therefore makes it more difficult for the evaluation software to find the correct frequency. However, an increase in damping occurred in the high-purity alumina samples from $1200^{\circ} \mathrm{C}$ onwards as well. In this case, softening of the grain boundaries could lead to an increase in attenuation.

As already described the application of the strongest frequency leads to gaps or jumps, which do not correlate with the material behavior. Taking into account all recorded frequencies, these gaps are closing. However, this evaluation method also increases the number of measurement points, which cannot be assigned to the main frequency. Due to the geometry deviating from a rectangular bar, at least one further flexural frequency occurs resulting from the surfaces rotated by $90^{\circ}$ along the longitudinal axis. Further frequency signals occur off the (main) curve. These signals are not reproducible in other measurements. Moreover, many of these frequencies are isolated. This means that they appear and vanish abruptly. They can usually only be followed for a very short time. Therefore, we consider that these are false measurements, caused by interference frequencies.

Particularly during the isothermal holding time, the individual measurement curves show a deviation of $1-2 \%$, so that the line becomes more of a measurement range. The evaluation showed that several relatively close frequencies were found in this range for one measurement time. In this case, this deviation is caused by the measurement inaccuracy. Within its scope, the evolution of the frequency can be reproduced with further samples. This allows to verify temperature ranges where the measurement signal may become worse. Most characteristic points of the curve match to corrosion phenomena mentioned in literature. The curves' progression generally agrees well with the common concepts. Therefore, it is in principle possible to use the RFDA to observe the corrosion course in-situ. However, due to the measurement inaccuracy, each experiment should be verified several times. In 
addition, the usual post mortem analyses should still be performed in order to evaluate the results. To consider further fundamental mechanisms, it is recommended to carry out additional experiments with well-characterized, highly pure and homogeneous materials as well as corrosion agents to confirm the influence on the elastic properties.

\section{Conclusions}

In order to follow the corrosion process of refractories in-situ, the frequency was measured using HT-RFDA. In previous studies, it was not possible to link characteristic features of the frequency versus time or temperature curves with specific corrosion mechanisms. To interpret the measurement signal, specific phenomena such as melting were investigated on simplified systems to exclude further disturbance factors and uncertainties and obtain unaffected results. This work has shown that typical phenomena during corrosion can be well linked to the recorded curves. The kinetics of the corrosion process could be tracked by means of the HT-RFDA. The measurement of the frequency change during corrosion is therefore a very promising in-situ measurement technique to investigate the process in detail. Especially isolated observation of single mechanisms on a model system is important to interpret the curves completely and correctly. However, it does not subsidize the usual post mortem analysis, but allows a better evaluation of the mechanisms during the corrosion process. In combination, it can be a powerful tool for understanding how materials interacts with corrosive media.

Author Contributions: Conceptualization, T.T., S.E. and W.R.; methodology, J.N. and W.R.; validation, J.N, P.L. and W.R.; investigation, E.K., J.N, P.L. and W.R.; writing—original draft preparation, J.N. and W.R.; writing-review and editing, T.T. and S.E.; visualization, J.N. and W.R.; supervision, R.T. and T.T.; project administration, J.N. and W.R. All authors have read and agreed to the published version of the manuscript.

Funding: This research received no external funding.

Acknowledgments: Special thanks goes to Alpha Ceramics, Alteo, BASF and Imerys for the friendly provision of raw materials.

Conflicts of Interest: The authors declare no conflict of interest.

\section{References}

1. Salmang, H.; Scholze, H. Keramik, 7th ed.; Telle, R., Ed.; Springer: Berlin/Heidelberg, Germany, 2007.

2. Winston Revie, R. Uhlig's Corrosion Handbook, 3rd ed.; John Wiley \& Sons, Inc.: Hoboken, NJ, USA, 2011.

3. DIN, German Institute for Standardization. Methods of Test for Dense Refractory Products—Guideline for Testing Corrosion of Refractories Caused by Liquids; German Version CEN/TS 15418:2006; Materials Testing Standards Committee: Berlin, Germany, 2006. [CrossRef]

4. Schacht, C. Refractories Handbook; CRC Press: Boca Raton, FL, USA, 2004.

5. ASTM International. C1548-02(2012) Standard Test Method for Dynamic Young's Modulus, Shear Modulus, and Poisson's Ratio of Refractory Materials by Impulse Excitation of Vibration; ASTM International: West Conshohocken, PA, USA, 2012. [CrossRef]

6. Andreev, K.; Tadaion, V.; Zhu, Q.; Wang, W.; Yin, Y.; Tonnesen, T. Thermal and mechanical cyclic tests and fracture mechanics parameters as indicators of thermal shock resistance: Case study on silica refractories. J. Eur. Ceram. Soc. 2019, 39, 1650-1659. [CrossRef]

7. Sibil, A.; Erauw, J.P.; Cambier, F.; R'Mili, M.; Godin, N.; Fantozzi, G. Study of damage of high zirconia fused-cast refractories by measurement of Young's modulus. Mater. Sci. Eng. A 2009, 521, 221-223. [CrossRef]

8. Traon, N.; Tonnesen, T.; Telle, R. Comparison of the elastic properties determined by different devices in a refractory castable based on partially stabilized zirconia. In Proceedings of the 53rd International Colloquium on Refractories, Aachen, Germany, 8-9 September 2010.

9. Tonnesen, T.; Telle, R. Evaluation of Thermal Shock Damage in Castables by a Resonant Frequency and Damping Method. In Proceedings of the 49th International Colloquium on Refractories, Aachen, Germany, 7-8 November 2006; pp. 133-136.

10. Etzold, S.; Traon, N.; Tonnesen, T.; Telle, R. Elastic and Mechanical Fatigue at High Temperatures of High-Alumina Castables with Addition of Partially Stabilized Zirconia. Interceram 2016, 65, 19-23. [CrossRef] 
11. Traon, N.; Schnieder, J.; Villalba, A.; Tonnesen, T.; Telle, R.; Huger, M.; Chotard, T. Influence of Andalusite, $\mathrm{Al}_{2} \mathrm{O}_{3}-\mathrm{ZrO}_{2}-\mathrm{SiO}_{2}$ and $\mathrm{Al}_{2} \mathrm{O}_{3}-\mathrm{ZrO}_{2}$ Addition on Elastic and Mechanical Properties of High Alumina Castables. Interceram-Int. Ceram. Rev. 2014, 63, 290-294. [CrossRef]

12. Traon, N.; Schnieder, J.; Tonnesen, T.; Telle, R.; Huger, M.; Chotard, T.; Belrhiti, Y.; Villalba, W.A. High Temperature Evaluation of Mechanical Properties of Refractory Castables. Refract. Worldforum 2017, 9, 116-126.

13. Reichert, W.; Nießen, J.; Kröll, E.; Tonnesen, T.; Telle, R.; Leto, P.; Haubner, R. In-situ Thermo-Elastic Measurements of High-Alumina Castables During Corrosion Process. In Proceedings of the 61st International Colloquium on Refractories, Aachen, Germany, 26-27 September 2018; ECREF: Aachen, Germany, 2018.

14. Tonnesen, T.; Reichert, W.; Telle, R.; Leto, P.; Haubner, R. Progess of Young's Modulus of High Alumina Castables during Corrosion Process. In Proceedings of the UNITECR 19, Yokohama, Japan, 13-16 October 2019.

15. Kyrillis, S.; Tonnesen, T.; Telle, R. Monitoring the elastic properties of a alumina based monolithics exposed to steel slag and the effect of preformed spinel addition. In Proceedings of the 62nd International Colloquium on Refractories, Aachen, Germany, 25-26 September 2019; ECREF: Aachen, Germany, 2019.

16. Hönig, S.; Koch, D.; Weber, S.; Etzold, S.; Tonnesen, T.; Telle, R.; Traon, N. Evaluation of dynamic modulus measurement for C/C-SiC composites at different temperatures. Int. J. Appl. Ceram. Technol. 2019, 16, 1723-1733. [CrossRef]

17. Roebben, G.; Bollen, B.; Brebels, A.; Van Humbeeck, J.; Van der Biest, O. Impulse excitation apparatus to measure resonant frequencies, elastic moduli, and internal friction at room and high temperature. Rev. Sci. Instrum. 1997, 68, 4511-4515. [CrossRef]

18. Wachtman, J.B., Jr.; Lam, D.G., Jr. Young's Modulus of Various Refractory Materials as a Function of Temperature. J. Am. Ceram. Soc. 1959, 42, 254-260. [CrossRef]

19. Manasijević, I.; Balanović, L.; Grgurić, T.H.; Minić, D.; Gorgievski, M. Study of Microstructure and Thermal Properties of the Low Melting Bi-In-Sn Eutectic Alloys. Mater. Res. 2018, 21. [CrossRef]

20. Carter, C.B.; Norton, M.G. Ohio Library and Information Network. In Ceramic Materials: Science and Engineering, 2nd ed.; Springer: New York, NY, USA, 2007.

(C) 2020 by the authors. Licensee MDPI, Basel, Switzerland. This article is an open access article distributed under the terms and conditions of the Creative Commons Attribution (CC BY) license (http://creativecommons.org/licenses/by/4.0/). 